Check for updates

Cite this: RSC Adv., 2017, 7, 55360

Received 9th November 2017

Accepted 30th November 2017

DOI: 10.1039/c7ra12267e

rsc.li/rsc-advances

\section{Preparation and characterization of a microemulsion via polycondensation of alkoxyl silane}

\author{
Liu Jiesheng, (D)* Gong Xiaoqiang, He Xiang and Zhang Rongtang
}

A microemulsion was successfully prepared via polycondensation of alkoxy silanes. Research into the control of the preparation and properties of the microemulsion was conducted. The structure and properties of the microemulsion were characterized by transmission electron microscopy (TEM) and photon correlation spectroscopy (PCS). The variation in the temperature, the methods, and the amount of catalyst and surfactant was studied in relation to the properties of the final microemulsion.

\section{Introduction}

Since the 1950's organo-functionalized silanes have attracted great interest as heat-resistant, low toxicity and environmentally stable materials for commercial applications. ${ }^{1}$ Their general formula is $\mathrm{Y}-\mathrm{Si}(\mathrm{OR})_{n}$, where $\mathrm{Y}$ is a functionalized organic substituent and OR stands for an alkoxy group. Organofunctional alkoxysilanes readily undergo hydrolysis in the presence of water and a catalyst. The hydrolytic reaction and polycondensation of alkoxysilanes $\mathrm{RSi}(\mathrm{OR})_{n}$ leads to the formation of several products useful in the synthesis of functionalized organic-inorganic hybrid materials. ${ }^{2}$ One of the most common applications of alkoxysilanes is in coatings. With environmental criteria becoming stricter, more and more drastic regulations aiming to reduce the emissions of VOCs (volatile organic compounds) into the atmosphere have led to water-based solvent paints and water borne coating emulsions gaining importance in the modern coating industry. ${ }^{3}$ In recent years, microemulsions have received considerable interest because of their small droplet size and complex structural behavior. ${ }^{4-8}$ Microemulsions are a low-viscosity, transparent, isotropic, and thermodynamically stable liquid medium. In previous reports on microemulsion, most studies referred to the addition of cosurfactants to help stabilize the microemulsion and employing free radical polymerization method to functionalize the system. However, these methods are often either less sensitive or have their intrinsic disadvantages, e.g., technical complications, time consuming processes or demanding that restricted available reagents. Thus, the development of new routes on preparation of microemulsions is considered to be one of the most challenging fields.

School of Civil Engineering and Architecture, Wuhan Polytechnic University, Wuhan, 430023, China. E-mail: wsljs628@yahoo.com; Fax: +86-027-83950207; Tel: +86027-83950207
In this paper, we describe a pathway to functional organosilicon microemulsions, which are prepared with alkoxysilane via hydrolysis and polycondensation. It is well known that the hydrolysis and condensation reaction of organofunctional alkoxysilane occurs in coating formulations and the polysiloxane with network structure and long siloxane chains was prepared. However, due to the nature of its long chain and the rigidity of 3D network backbone structure, the polysiloxane microemulsions are more prone to coalescence, separation, and thus are poor stability and poor processibility. ${ }^{9}$ According to the traditional methods to stabilize the microemulsion, the cosurfactant would be added. However, compared to those microemulsions composed of water, oil, surfactant, and coemulsifier, the microemulsion prepared in this work was prepared in the presence of water, monomer, a surfactant and without co-emulsifier. A catalyst, $\mathrm{C}_{12} \mathrm{H}_{25} \mathrm{SO}_{3} \mathrm{H}$, (DBSA), as surfactant-like species was employed without further treatment.

Based on the aforementioned considerations, our aim is to investigate the control over the preparation and properties of microemulsion. To the best of our knowledge, this research has likewise not been reported on previously. The variation in catalyst, emulsifier and monomer dropping rate on the properties of microemulsion were also discussed.

\section{Experimental}

\subsection{Materials}

Alkoxy silanes, $\gamma$-(2,3-epoxypropoxy)propyltrimethoxysilane (KH560) and methacryloxypropyl trimethoxylsilane (KH570), purchased from Hubei province of China, was used without any further purification. A nonionic surfactant, (OP-10), used without any further purification, $>95 \% ; \mathrm{pH}=5-7$. Dodecyl benzenesulfonic acid, $\left(\mathrm{C}_{12} \mathrm{H}_{25} \mathrm{C}_{6} \mathrm{H}_{4} \mathrm{SO}_{3}\right)$, DBSA, purchased from Henan province in China, acts as catalyst, industrial material; $\mathrm{pH}=0.4 ; \mathrm{mp} 16.3{ }^{\circ} \mathrm{C}$; b.p. $286{ }^{\circ} \mathrm{C} / 760 \mathrm{mmHg}$. Water was 
distilled and deionized before using, $\mathrm{pH}=5.0-7.0$; electrical conductivity $\leq 0.5 \mu \mathrm{s} \mathrm{cm}^{-1}\left(25^{\circ} \mathrm{C}\right)$; specific resistivity $\geq 2 \mathrm{M} \Omega$.

\subsection{Preparation of microemulsion}

Catalyst, non-ionic emulsifier and distilled water were placed in a four-neck round bottom flask equipped with a mechanical stirrer, reflux condenser, thermometer, and nitrogen gas inlet. KH560 and KH570 were mixed with the weight ration of $1: 1$ as alkoxy silanes compound. Then, the alkoxy silanes compound was dropped into the flask. The polymerization was continued thereafter in batch for 4 hours and the microemulsions formed.

\subsection{Characterization}

The particle size and size distribution were characterized by PCS, which was made in Malvern Company in U.K. The particle configuration was exhibited by TEM, made in Japan.

\section{Results and discussion}

\subsection{Effect of the temperature on the microemulsion}

Table 1 shows the effect of the reaction temperature on the microemulsion. As seen in Table 1 that the temperature greatly affect the particle size and size distribution of the microemulsion. It was observed that the particle size diameter decreased with increase in the reaction time from $35^{\circ} \mathrm{C}$ to $45^{\circ} \mathrm{C}$. The particle size diameter of microemulsion prepared with the reaction temperature $35{ }^{\circ} \mathrm{C}$ is around $88.2 \mathrm{~nm}$. With increase of the reaction temperature to $45{ }^{\circ} \mathrm{C}$, the particle size of the microemulsion decreases to around $51 \mathrm{~nm}$. However, a significant increase in the size distribution of microemulsion was observed with increase in reaction temperature. Since the hydrolysis of the alkoxy silane and the polycondensation were found to be temperature sensitive. At the higher temperatures, the rate of hydrolysis reaction is rather higher. More active

Table 1 Effect of the reaction temperature on the microemulsion

\begin{tabular}{lll}
\hline Reaction temperature $/{ }^{\circ} \mathrm{C}$ & Particle size $/ \mathrm{nm}$ & Size distribution \\
\hline 35 & 88 & 0.026 \\
40 & 65 & 0.032 \\
45 & 51 & 0.12
\end{tabular}

oligomer active centers occur and the rate of polymerization increases and the reactions among the particles become vicious making the particle size decrease and the size distribution broaden. This can be proved in Fig. 1. Fig. 1(a-c) shows the morphology of the microemulsion with different reaction temperature. As seen in Fig. 1(a) that the particles of microemulsion with $35{ }^{\circ} \mathrm{C}$ are in equal dimensions and the particle size is around $90 \mathrm{~nm}$. Increase of the temperature decreases the particle size and produces microemulsion of variable droplet size as shown in Fig. 1(b). In Fig. 1(c), the average particle size of microemulsion with $45{ }^{\circ} \mathrm{C}$ decreases to $50 \mathrm{~nm}$ and most particles are not of uniform size. According to Kim et al. ${ }^{10}$ low polymerization rates can yield highly ordered nano-structures with fewer structural defects. Thus, the reaction temperature of $35^{\circ} \mathrm{C}$ was chosen for the preparation of the microemulsion in this study.

\subsection{Effect of the catalyst on the microemulsion}

Fig. 2 presents the morphology for microemulsion with different amounts of catalyst. The effect of catalyst on the particle size and size distribution was observed. As seen in Fig. 2(a) and (b) that the size distribution get higher as the amount of catalyst increases, while the particle size presents downtrend. For instance, microemulsions with $1 \%$ catalyst, the mean particle diameter is around $101 \mathrm{~nm}$ and the particle size decreases to $82.8 \mathrm{~nm}$ and a small tendency to agglomeration occurs when the content of catalyst goes up to $3.0 \%$ of the microemulsion. The effect of catalyst with lower amount $(<5 \%)$ has a similar effect on the particle size and size distribution studied as that obtained in the microemulsion with different reaction temperature. The catalyst, DBSA, a surfactant-like species, which contains hydrophilic $\left(-\mathrm{SO}_{3} \mathrm{H}\right)$ and hydrophobic $\left(\mathrm{C}_{12} \mathrm{H}_{25^{-}}\right)$functional groups, acts as catalysis and emulsification roles. ${ }^{11}$ The increase in DBSA content results in the acidity strengthened and makes the number of oligomer active centers increase. Under strong acidic condition, the rate of polymerization increases and the reactions among the particles become vicious making the size distribution broaden. As the content of DBSA increases, the surfactant effect of DBSA increases the average surfactant coverage of particles, and provides more micelles, which makes the particle size lessen. However, it can be noted that further increase in the catalyst amount makes the acidity of the microemulsion strengthened and results in the
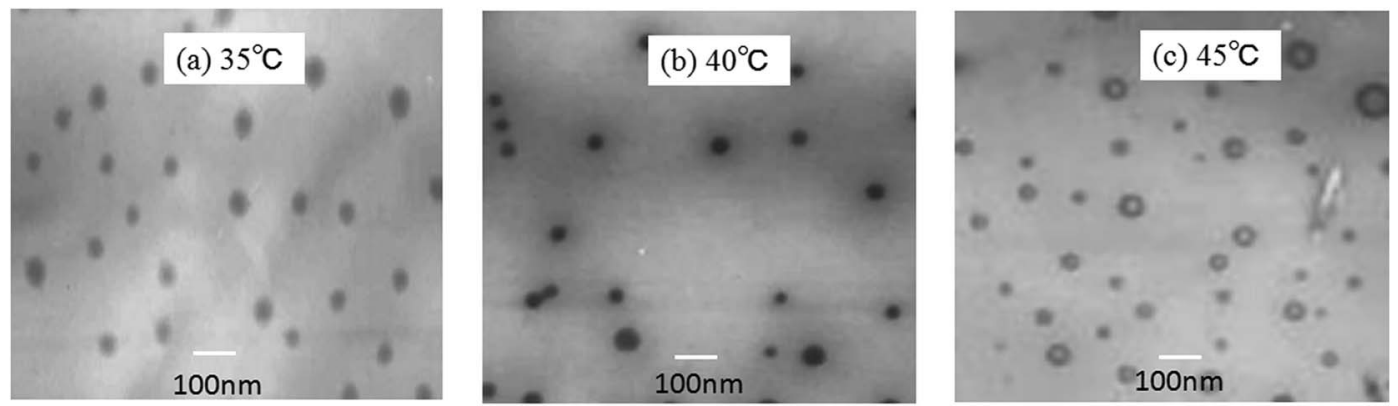

Fig. 1 TEM images of microemulsions prepared with different reaction temperature. 

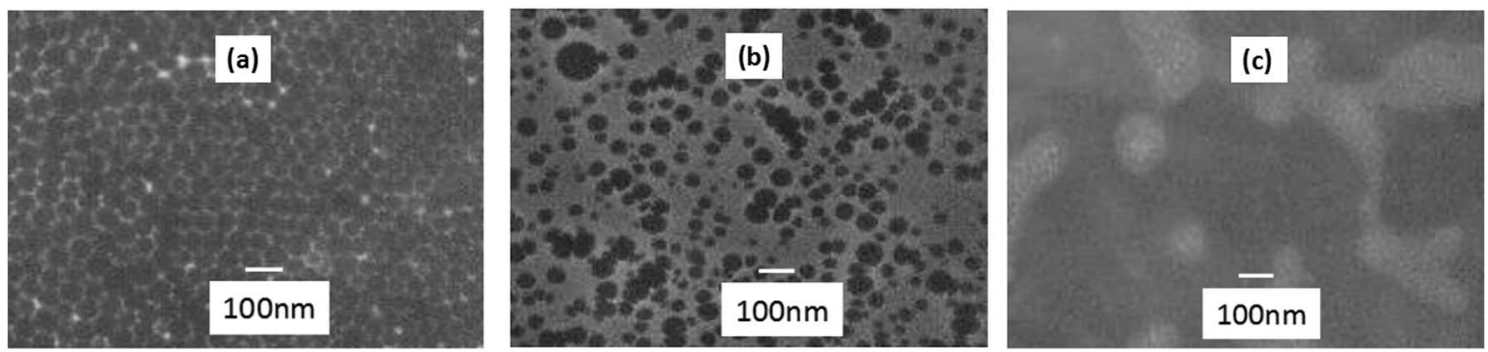

Fig. 2 TEM images of microemulsions prepared with different catalyst content.

instability of microemulsions, as shown in Fig. 2(c). Thus, it can be concluded that the catalyst has pronounced effect on the stabilization/breaking of emulsion droplet. The microemulsions show stable properties when the amount of catalyst is below $5.0 \mathrm{wt} \%$.

\subsection{Effect of the emulsifier on the microemulsion}

Table 2 shows the effect of catalyst on the particle size and size distribution of microemulsion. The size distribution get higher as the amount of emulsifier increases, while the particle size presents downtrend. For instance, the mean diameter of the particles of microemulsion with $1 \%$ OP-10 is $87.5 \mathrm{~nm}$ and its polydispersity reaches 0.015 , which means that the sample is rather homogeneous. The particle size reduced to $62.4 \mathrm{~nm}$ when the content of emulsifier goes up to $3.0 \%$ of the microemulsion and the size distribution increases to 0.104 . This phenomenon can be further confirmed by configuration in TEM images.

TEM imagines of the microemulsions, presented in Fig. 3, were collected with different amount of emulsifier. As seen in Fig. 3(a), the particles show sparse and homogeneous configuration. The increase in the OP-10 amount makes the particle in varied size and the average size decrease. In addition, the particles show more dense configuration when the emulsifier is

Table 2 Effect of emulsifier on the microemulsion

\begin{tabular}{lll}
\hline Amount of OP-10/\% & Particle size $/ \mathrm{nm}$ & Size distribution \\
\hline 1 & 87 & 0.015 \\
2 & 78 & 0.072 \\
3 & 62 & 0.104
\end{tabular}

at higher content. It is likely explained by the reason that more micelles were produced when the content of emulsifier is higher, which favored the dispersion of more active species. When the emulsifier content was lower, there are fewer amounts of particles and the particles are prone to grow on the quondam molecular chain, which results in the larger particle size and wider size distribution. As the amount of emulsifier increases, more particles were produced. Condensation reaction acting among the particles, results in the smaller particle size and wider size distribution. ${ }^{12}$

\subsection{Effect of the dropping rate on the microemulsion}

The preparation method has an important role on the structure and properties of the microemulsion. Two methods were applied for the synthesis: one time joining method and monomer was added dropwise at a speed of $1 \mathrm{ml} \mathrm{min}^{-1}$. Table 3 shows the effect of dropping rate of monomer on the microemulsion. As seen in Table 3, the mean diameter of the particles of microemulsion with one time joining method is $121 \mathrm{~nm}$ and its polydispersity reaches 0.015 , which means that the sample is rather homogeneous. However, the particle size decreased to $68 \mathrm{~nm}$ and the size distribution increased to 0.064. This phenomenon can be further illustrated in SEM images.

Table 3 Effect of dropping rate of monomer on the microemulsion

\begin{tabular}{|c|c|c|}
\hline Methods & $\begin{array}{l}\text { One time joining } \\
\text { method }\end{array}$ & $\begin{array}{l}\text { Monomer added } \\
\text { dropwise method }\end{array}$ \\
\hline Particle size (nm) & 121 & 68 \\
\hline Size distribution & 0.015 & 0.064 \\
\hline
\end{tabular}
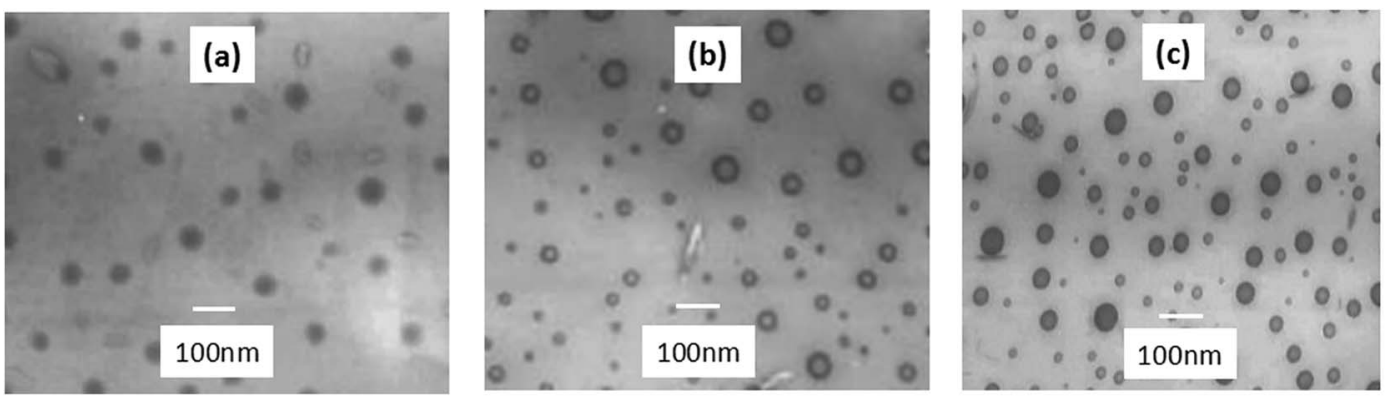

Fig. 3 TEM images of microemulsions prepared with different emulsifier content. 

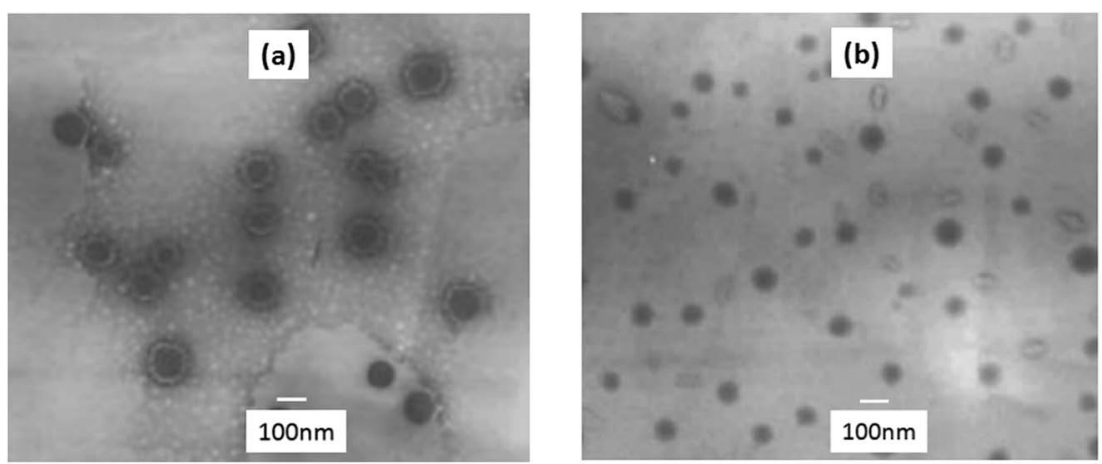

Fig. 4 TEM images of microemulsion prepared with different methods.

Fig. 4 shows the TEM images of microemulsion with different methods. Compared with batch addition (Fig. 4(a)), the particle size of latex by monomer drop-wise was smaller and the size distribution was wider (Fig. 4(b)). A likely explanation was related to the concentration of active silane in spacing interval. When the monomer was added by drop-wise, it is easily to form emulsion particles in different size. The subsequent monomer dropped into the microemulsion, reacted with the varisized particles, which result in the smaller particle size and wider size distribution. Due to the control in the particle size and the stability of the microemulsion, the drop-wise addition of alkoxy silanes was chosen for the microemulsion preparation in this study.

\section{Conclusions}

The main purpose of this study has been to carry out an experimental investigation to the control in the preparation and properties of the microemulsion via hydrolysis and polycondensation of alkoxyl silanes. In light of the obtained results, the following are the main conclusions of the present work:

(1) Microemulsion was successfully prepared via polycondensation of alkoxy silanes under acidic condition.

(2) Higher temperature decreases the particle size, produces microemulsion of variable droplet size and makes the microemulsion unstable. The reaction temperature of $35{ }^{\circ} \mathrm{C}$ was chosen for the preparation of the microemulsion.

(3) The catalyst has pronounced effect on the stabilization/ breaking of emulsion droplet. The microemulsions show stable properties when the amount of catalyst is below $5.0 \mathrm{wt} \%$.

(4) The increase in the emulsifier amount makes the particle in varied size and the average size decrease.

(5) Compared with batch addition, the particle size of latex by monomer drop-wise was smaller and the size distribution was wider. Due to the control in the particle size and the stability of the microemulsion, the drop-wise addition of alkoxy silanes was chosen for the microemulsion preparation in this study.

\section{Conflicts of interest}

There are no conflicts to declare.

\section{Acknowledgements}

This work was supported by the National Natural Science Foundation of China (51409203), and the writers would like to express their great appreciation of the funding supported by National Natural Science Foundation of Hubei Province in China (2017CFB510) and State Key Laboratory of Silicate Materials for Architectures (Wuhan University of Technology) (SYSJJ2016-13).

\section{References}

1 P. H. Michael, K. Steffen and B. Heinz, Int. J. Adhes. Adhes, 2016, 64, 153.

2 B. S. Marie-Christine and N. B. Mohamed, Colloids Surf., A, 2010, 366, 147.

3 R. Palkovits, H. Althues, A. Rumplecker and B. Tesche, Langmuir, 2005, 21, 6048.

4 M. Bonini, U. Bardi, D. Berti, C. Neto and P. Baglioni, J. Phys. Chem. B, 2002, 106, 6178.

5 J. J. Shrikhandea, P. A. Hassanb and R. V. Jayarama, Colloids Surf., A, 2010, 370, 64.

6 V. Castellino, Y. L. Cheng and E. Acosta, J. Colloid Interface Sci., 2011, 353, 196.

7 S. A. Abdullah, A. A. Zeid, O. Mohamed and A. Taieb, Desalination, 2010, 257, 87.

8 P. G. Ronald, Colloids Surf., A, 1998, 137, 91.

9 J. MacMullen, Z. Y. Zhang, J. Radulovic, H. N. Dhakal and N. Bennett, Constr. Build. Mater., 2012, 37, 283.

10 B. J. Kim, S. G. Oh, M. G. Han and S. S. Im, Synth. Met., 2001, 122, 297.

11 L. Jiesheng, H. Shiqiang and L. Donglai, Iran. Polym. J., 2011, $10,825$.

12 L. Jiesheng, W. Shaopeng, H. Shiqiang, C. Peiliang and M. Yixuan, Iran. Polym. J., 2009, 2, 159. 